In vivo confocal microscopy indicates an inverse relationship between the subbasal corneal plexus and the conjunctivalization in patients with limbal stem cell deficiency (see page 327 ) In this study the authors observed an inverse relationsip between sub-basal corneal nerve plexus density and conjunctivalisation, providing some insight into the aetiopathogenesis of limbal stem cell deficiency.

Lack of tumour pigmentation in conjunctival melanoma is associated with light iris colour and worse prognosis (see page 332)

The authors found an association between low tumour pigmentation, light iris colour and a worse clinical outcome with a greater risk for recurrences, metastasis and death in patients with conjunctival melanoma.

Rapid assessment of avoidable blindness in Papua New Guinea: a nationwide survey (see page 338)

Papua New Guinea has one of the highest prevalences of blindness and vision impairment globally. This high prevalence is related to limited access to cataract surgery, and refractive error services.

Seasonal variation of refractive error change among young schoolchildren in a population-based cohort study in Taipei (see page 343 )

Seasonal variation in refractive error change exists among young schoolchildren living in a subtropical city and is more pronounced in nonmyopic than in myopic schoolchildren.

Intraocular pressure and myopia progression in Chinese children: the Anyang childhood eye study (see page 349)

In this longitudinal study, children with faster myopia progression had lower baseline IOP than children with slower myopia progression. This inverse relation between IOP and myopia progression supports the hypothesis that high IOP is not the initiating factor for myopia development.

Ten-year incidence of primary angleclosure in elderly Chinese: the Liwan eye study (see page 355 )

In this 10 year follow-up study, the cumulative incidence of primary angle closure in phakic eyes was $20.5 \%$ (127/620), including $16.9 \%, 2.4 \%$ and $1.1 \%$ for incident PACS, $\mathrm{PAC}$ and PACG, respectively.
Variability of vertical cup-disc ratio measurement and the effects of glaucoma 5 -year risk estimation in untreated ocular hypertensive eyes (see page 361 )

The article investigate how different methods of vertical cup-to-disc measurement could affect the risk calculation for patients with ocular hypertension.

The impact of SIGN glaucoma guidelines on false positive referrals from community optometrists in central Scotland (see page 369)

The Scottish Intercollegiate Guidelines Network (SIGN) guideline 144 was introduced to improve glaucoma referral quality. The authors observed a significant reduction in the first visit discharge rate after guideline implementation.

Optic nerve head cupping in glaucomatous and Nonglaucomatous optic neuropathy (see page 374 )

Anterior laminar depth is deeper in glaucoma than in a subset of non glaucomatous cupping, such as compressive optic neuropathy, methanol optic neuropathy, optic neuritis, and periventricular leukomalacia.

Diagnostic value of ganglion Cell-Inner plexiform layer for early detection of ethambutol-induced optic neuropathy (see page 379 )

Ganglion cell-inner plexiform layer analysis was observed to provide a good diagnostic tool. The authors suggest a clinical guideline for early detection of challenging cases of ethambutol optic neuropathy.

Comparison of long-term clinical evolution in highly myopic eyes with vertical oval-shaped dome with or without untreated serous retinal Detachment (see page 385)

This prospective study of 40 highly myopic eyes with vertical oval-shaped domes demonstrated that untreated patients with serous foveal retinal detachment seem to remain stable over time in terms of visual function.

Detailed genetic characteristics of an international large cohort of patients with Stargardt disease: ProgStar Study report \#8. (see page 390)

This study addresses 211 ABCA4 mutations in the largest cohort of 345 patients with Stargardt disease. Half of the cohort harbours only missense variants, in keeping with the relatively mild phenotype of the cohort.

Contrast sensitivity and visual acuity under low light conditions in macular telangiectasia type 2 (see page 398 )

Contrast sensitivity is considerably impaired under low light conditions in early disease stages of MacTel. It might aid earlier detection of the condition and also be useful as an additional outcome measure in interventional trials.

Macular dysfunction in patients with macula-on rhegmatogenous retinal detachments (see page 404)

Focal ERG responses from the macula of eyes with macula-on rhegmatogenous retinal detachment suggested macular dysfunction, showing significant reduction in the amplitude of a- and b-waves and oscillatory potentials in comparison with the fellow eyes.

Acute macular neuroretinopathy: pathogenetic insights from optical coherence tomography angiography (see page 410)

Optical coherence tomography angiography in acute macular neuroretinopathy demonstrates global reduction of the vascular flow in the inner choroid and reduced vessel density of the deep capillary plexus limited to the extension of the lesion.

Repeatability, Interocular correlation and agreement of quantitative SweptSource optical coherence tomography angiography macular metrics in healthy subjects (see page 415)

This study investigated repeatability, the inter-eye correlation and agreement of SS-OCTA metrics in normal subjects. The results show excellent repeatability in macular measurement and the inter-eye correlation is high for FAZ area and perimeter, moderate for fractal dimension and VDI, while low for vessel density.

Lymphangiogenesis in failed corneal transplants with neovascularization (see page 421)

Human corneal graft failure with neovascularization is associated with lymphangiogenesis. 


\section{At a glance}

Diagnostic value of a combination of next-generation sequencing, chorioretinal imaging, and metabolic analysis: lessons from a consanguineous Chinese family with gyrate atrophy of the choroid and retina stemming from a novel variant (see page 448)

GACR was accurately diagnosed in a consanguineous Chinese family and a novel
OAT variant identified by NGS, chorioretinal imaging and metabolic analysis. This study will help to diagnose GACR disease and guide future disease treatment. 